\title{
Microarray pathway analysis indicated that mitogen-activated protein kinase/extracellular signal-regulated kinase and insulin growth factor 1 signaling pathways were inhibited by small interfering RNA against AT-rich interactive domain $1 \mathrm{~A}$ in endometrial cancer
}

\author{
YE YANG ${ }^{1}$, WEI BAO ${ }^{2}$, ZHENGYU SANG $^{2}$, YONGBING YANG ${ }^{2}$, MENG LU $^{2}$ and XIAOWEI XI ${ }^{1}$ \\ ${ }^{1}$ Department of Obstetrics and Gynecology, Shanghai General Hospital of Nanjing Medical University; ${ }^{2}$ Department of \\ Obstetrics and Gynecology, Shanghai General Hospital of Shanghai Jiaotong University, Shanghai 200080, P.R. China
}

Received March 11, 2017; Accepted October 20, 2017

DOI: $10.3892 / \mathrm{ol} .2017 .7489$

\begin{abstract}
Mutations in the gene encoding AT-rich interactive domain 1A (ARID1A) are frequently observed in endometrial cancer (EC) but the molecular mechanisms linking the genetic changes remain to be fully understood. The present study aimed to elucidate the influence of ARID1A mutations on signaling pathways. Missense, synonymous and nonsense heterozygous ARID1A mutations in the EC HEC-1-A cell line were verified by Sanger sequencing. Mutated ARID1A small interfering RNA was transfected into HEC-1-A cells. Biochemical microarray analysis revealed 13 upregulated pathways, 17 downregulated pathways, 14 significantly affected disease states and functions, 662 upstream and 512 downstream genes in mutated ARID1A-depleted HEC-1-A cells, among which the mitogen-activated protein kinase/extracellular signal-regulated kinase and insulin-like growth factor-1 (IGF1) signaling pathways were the 2 most downregulated pathways. Furthermore, the forkhead box protein $\mathrm{O} 1$ pathway was upregulated, while the IGF1 receptor, insulin receptor substrate 1 and phosphatidylinositol-4,5-bisphosphate 3-kinase catalytic subunit b pathways were downregulated. Carcinoma tumorigenesis, tumor cell mitosis and tumor cell death were significantly upregulated disease states and functions, while cell proliferation and tumor growth were significantly downregulated. The results of the present study suggested that ARID1A may be a
\end{abstract}

Correspondence to: Professor Xiaowei Xi, Department of Obstetrics and Gynecology, Shanghai General Hospital of Nanjing Medical University, 100 Haining Road, Shanghai 200080, P.R. China E-mail: xixiaowei1966@126.com

Key words: AT-rich interactive domain 1A, endometrial cancer, mitogen-activated protein kinase/extracellular signal-regulated kinase signaling pathway, insulin growth factor-1 signaling pathway, microarray pathway analysis potential prognostic and therapeutic molecular drug target for the prevention of EC progression.

\section{Introduction}

Endometrial cancer (EC) is one of the most common invasive types of gynecologic cancer and accounts for $40 \%$ of gynecological cancer cases worldwide in 2015 (1). High insulin levels and obesity are independent risk factors for estrogen-related type I endometrial endometrioid carcinomas (2). At present, surgery combined with adjuvant progesterone therapy or postsurgical radiotherapy is the predominant treatment strategy for endometrial cancer (3). However, certain patients also suffer cancer recurrence and metastasis, rendering the prognosis poor (3). For effective cancer prevention and treatment, it is necessary to identify critical genetic changes that initiate endometrial cancer and contribute to its progression (4). Previous genome-sequencing efforts have supported a strong genetic component of endometrial cancer based on the cBioPortal for Cancer Genomics database (http://www.cbioportal.org) and have revealed a high frequency $(\leq 33.3 \%)$ of tumor mutations in AT-rich interactive domain 1A (ARID1A), a key member of the mating type switching/sucrose non-fermenting chromatin-modeling complex (5).

ARID1A is located at chromosome 1p36.11, a region frequently deleted in human cancer and encodes a nuclear protein associated with chromatin remodeling. ARID1A is frequently mutated in multiple gynecologic tumors, particularly in endometrium-associated neoplasms, including 50\% of endometriosis-associated ovarian clear cells, $30 \%$ of endometrioid ovarian carcinomas and $20-30 \%$ of endometrial carcinomas, depending on the histological subtype (6-8). Mutations in the ARID1A gene usually result in a loss of expression of the ARID1A-encoded protein. Certain ARID1A gene mutations eliminate the expression of the ARID1A protein, producing abnormalities in chromatin remodeling and promoting the malignancy of multiple types of cancer. Silencing mutated ARID1A may represent an approach to the prevention or treatment of malignant tumors. 
However, previous studies have primarily provided insight into the function of wild-type $\operatorname{ARID1A}(9,10)$. Furthermore, in EC, the majority of previous studies have focused on the loss of ARID1A expression as determined by immunohistochemistry or gene sequencing of mutations (7-11). Therefore, it remains unclear which signaling pathways are influenced by ARID1A mutations and which pathway molecules are targeted by ARID1A mutations in EC pathophysiology. Therefore, the present study aimed to elucidate the mechanistic details underpinning the effects of ARID1A mutations on different signaling pathways.

\section{Materials and methods}

Cell culture. The human endometrial cancer HEC-1-A cell line was purchased from Shanghai GeneChem Co., Ltd. (Shanghai, China). The cells were maintained in Dulbecco's modified Eagle's medium (DMEM; Corning Incorporated, Corning, NY, USA) supplemented with $10 \%$ fetal bovine serum (VS500T; Ausbian; Vian-Saga Co., Ltd, Shanghai, China) at $37^{\circ} \mathrm{C}$ in $5 \%$ $\mathrm{CO}_{2}$. For transfections, cells were seeded onto a 6-well plate $24 \mathrm{~h}$ prior to the experiment at a cell density of $3-5 \times 10^{4} / \mathrm{ml}$ in DMEM in an atmosphere of $5 \% \mathrm{CO}_{2}$ at $37^{\circ} \mathrm{C}$. Each experiment was performed in triplicate.

Exon sequencing. ARID1A mutations in HEC-1-A cells were verified by Sanger sequencing using a 3730XL DNA analyzer (Thermo Fisher Scientific, Inc., Waltham, MA, USA) and specific primers targeting exons 1212, 3969, 5281 and 5503 of the ARID1A NM_006015 CDS region were used. The experimental workflow of the Sanger sequencing included the following 6 steps: Isolating the DNA, performing a polymerase chain reaction $\left(\mathrm{PCR}\right.$; incubate at $95^{\circ} \mathrm{C}$ for $10 \mathrm{~min}$, denature at $96^{\circ} \mathrm{C}$ for $3 \mathrm{sec}$, anneal at $58^{\circ} \mathrm{C}$ for $15 \mathrm{sec}$, extend at $72^{\circ} \mathrm{C}$ for $30 \mathrm{sec}$ and final extension at $72^{\circ} \mathrm{C}$ for $10 \mathrm{~min}$ for 35 cycles with DNA polymerase (BigDYe3.1; Applied Biosystems; Thermo Fisher Scientific, Inc.), then hold at $4^{\circ} \mathrm{C}$ ), performing a sequencing reaction, purifying the sequencing reaction, performing capillary electrophoresis and analyzing the data using Applied Biosystems sequence scanner software v2.0 (Thermo Fisher Scientific, Inc.). PCR forward and reverse primers were designed according to the ARID1A gene fragment downloaded from the NCBI website (https://www.ncbi.nlm.nih.gov/nuccore/NC_000 001.11? report $=$ genbank $\&$ from $=26696031 \&$ to $=26782110$ ) and (https://www.ncbi.nlm.nih.gov/tools/primer-blast/index. cgi?LINK_LOC=BlastHomeAd). Gene sequencing of HEC-1-A was performed using gene-specific primers. The following PCR primers designed for exon 1212 were amplified at 479 bp: GSPE91935-primer 1, GTTACTAGGTTGGTCTCATTGCTC and GSPE91935-primer 2, AGCCAACAGGTCTACATTCCT GTC. The following PCR primers designed for exon 3969 were amplified at 353 bp: GSPE91935-primer 3, TGAAGCTATAGT GGGCTCAATCTG and GSPE91935-primer 4, CTGTTG ATACATTGTAGTCTGCTG. The following PCR primers designed for exons 5281 and 5503 were amplified at 573 bp: GSPE91935-primer 5,TCCTTGTAGAATATTTCCGACGATG and GSPE91935-primer 6, GTTTTTCTGGAGGTCCATCAG GTG.

Lentiviral vector production. Three short hairpin RNA (shRNA) sequences were designed by Shanghai GeneChem
Co., Ltd. The shRNA expression cassettes were designed according to the small interfering (siRNA) sequences. The shRNA vectors were generated by inserting annealed oligo sequences into the digested GV248 vectors (Shanghai GeneChem Co., Ltd.) between the Phu6 and Pubi sites. HEC-1-A cells were infected with lentiviral vector GV248 or ARID1A-specific shRNA (Shanghai GeneChem Co., Ltd.) particles with a GV248 plasmid $\left(2 \mu 1\right.$ at $1 \times 10^{9} \mathrm{TU} / \mathrm{ml}$, $4 \mu \mathrm{l}$ at $5 \times 10^{9} \mathrm{TU} / \mathrm{ml}$ and $2 \mu \mathrm{l}$ at $\left.1 \times 10^{9} \mathrm{TU} / \mathrm{ml}\right)$ in 6 -well plates in the presence of $6 \mathrm{mg} / \mathrm{ml}$ polybrene (Genomeditech Co., Ltd., Shanghai, China) and were then treated with $2 \mathrm{mg} / \mathrm{ml}$ puromycin (Clontech Laboratories, Inc., Mountainview, CA, USA) to generate stable clones. An empty vector was used as a control. ARID1A gene expression and protein levels were confirmed by reverse transcription-quantitative polymerase chain reaction (RT-qPCR).

$R T$ - $q P C R$. To measure the abundance of ARID1A mRNA, primers were selected and tested at different primer concentrations. Total RNA was extracted from EC cells using the TRIzol (Thermo Fisher Scientific, Inc.) method. A reverse transcription kit (Promega Corporation, Madision, WI, USA) was used to reverse transcribe the RNA into cDNA at $42^{\circ} \mathrm{C}$ for $1 \mathrm{~h}$. Subsequently, qPCR was performed using SYBR-Green (DRR041B; Takara Biotechnology Co., Ltd., Dalian, China) and fluorescence microscopy (model no. IX71; Olympus Corporation, Tokyo, Japan), The ARID1A siRNA sequences were as follows: 5'-GTCCCTCAAGTCTGGTCTCC-3' and reverse, 5'-GATCTCAATCAGGCATCGTC-3'. The thermocycling conditions were as follows: Degeneration at $95^{\circ} \mathrm{C}$ for $30 \mathrm{sec}$, annealing at $60^{\circ} \mathrm{C}$ for $30 \mathrm{sec}$ and extension at $72^{\circ} \mathrm{C}$ for $1 \mathrm{~min}$, for 40 cycles. Relative gene expression levels were calculated using the $2^{-\Delta \Delta c q}$ method (12) and were normalized to the expression of GAPDH (forward, 5'-TGACTTCAACAG CGACACCCA-3' and reverse, 5'-CACCCTGTTGCTGTA GCCAAA).

Western blot analysis. The transfected cells were washed twice with phosphate-buffered saline, centrifuged at $4^{\circ} \mathrm{C}, 1,000 \mathrm{x} \mathrm{g}$ for $5 \mathrm{~min}$ and lysed on ice in radio immunoprecipitation assay (RIPA) lysis buffer (WB-0071; Thermo Fisher Scientific, Inc.) containing protease inhibitors for $30 \mathrm{~min}$. Subsequently, the protein was quantified using the bicinchoninic acid method with BCA protein assay kit (P0010S; Beyotime Institute of Biotechnology, Haimen, China). The protein (20 $\mu \mathrm{g}$ per lane) was subjected to SDS-PAGE electrophoresis to separate the protein using a 5\% stacking gel and $10 \%$ separating gel, prior to being transferred onto polyvinylidene fluoride membranes. Membranes were blocked by Tris-buffered saline containing $0.05 \%$ Tween-20 (TBST) and 5\% skimmed milk for $1 \mathrm{~h}$ at room temperature and were incubated overnight with one of the following primary antibodies for $30 \mathrm{~min}$ at $4{ }^{\circ} \mathrm{C}$ : Anti-rabbit eukaryotic translation initiation factor 4E (EIF4E; cat. no. 2067; dilution, 1:500; Cell Signaling Technology, Inc., Danvers, MA, USA), anti-FOS (cat. no. 8333; dilution, 1:500; Cell Signaling Technology, Inc.), anti-rabbit insulin receptor substrate 1 (IRS1; cat. no. ab52167; dilution, 1:500; Abcam, Cambridge, MA, USA), anti-rabbit phosphatidylinositol-4,5-bisphosphate 3-kinase catalytic subunit $\beta$ (PIK3CB; cat. no. ab32569; dilution, 1:500; Abcam), anti-rabbit forkhead 
box protein O1 (FOXO1; cat. no. 2880; dilution, 1:500; Cell Signaling Technology, Inc.) and anti-rabbit insulin-like growth factor 1 receptor (IGF1R; cat. no. ab131476; dilution, 1:500; Abcam). Following washing, the horseradish peroxidase-conjugated goat anti-rabbit IgG secondary antibody (cat. no. sc-2004; dilution, 1:2,000; Santa Cruz Biotechnology, Inc., Dallas, TX, USA) was added for $2 \mathrm{~h}$ at room temperature. The anti-mouse GAPDH (cat. no. sc-32233; dilution, 1:2,000, Santa Cruz Biotechnology, Inc.) was used as a sample loading control. Pierce ${ }^{\mathrm{TM}}$ ECL Western Blotting Substrate kit (Thermo Fisher Scientific, Inc.) was used for enhanced chemiluminescence, and the membranes were then placed on X-ray film (Carestream, Health, Inc., Rochester, NY, USA). The X-ray film was placed in X-ray film imaging powder (P61-04-1; Guanlong, Shanghai, China) for $1 \mathrm{~min}$ and then in X-ray film fixing powder (Guanlong, Shanghai, China) for $2 \mathrm{~min}$. The data were normalized to GAPDH expression by densitometry. Western blot automated quantitative analysis system Compass software (version WS-2471; Protein Simple, San Jose, CA, USA) was used to analyze the gradation test results. Quantitative results, including molecular weight, signal intensity (area), \% area and signal-to-noise ratio were obtained for each immunodetected protein.

Microarray pathway analysis (PathArray ${ }^{T M}$ ) using Ingenuity ${ }^{\circledR}$ Pathway Analysis $\left(I P A^{\circledR}\right)$. Human Gene Chip Prime View (cat. no. 901838; Affymetrix; Thermo Fisher Scientific, Inc.) was used to simultaneously investigate alternations in the activities of canonical signaling pathways in response to ARID1A depletion. The relative activity of each pathway was determined and normalized to that of untreated controls. Experiments were performed in triplicate and the values were calculated as the mean \pm standard error of the mean. The sample quality was required to meet the following standards: NanoDrop 2000 (Thermo Fisher Scientific, Inc.), 1.7<A260/A280<2.2; Agilent 2100 Bioanalyzer (Agilent Technologies, Inc., Santa Clara, CA, USA), RNA integrity number $\geq 7.0$; and $28 \mathrm{~S} / 18 \mathrm{~S}>0.7$. Expression data were normalized through quantile normalization. All gene level files were imported into IPA ${ }^{\circledR}$ software (v39473382; Qiagen $\mathrm{GmbH}$, Hilden, Germany; http://www.ingenuity.com) for further analysis.

The distributions of the intensities of 6 samples and the similarities between ARID1A-knockdown (KD) and control (NC) groups were examined by three-dimensional principal component analysis (Fig. 1A) and Pearson's correlation of the signal value (Pearson's correlation coefficient $>0.95$; Fig. 1B). Signal value distribution (Fig. 1C) and relative signal box plot graphs (Fig. 1D) demonstrated the expression values of all microarray probe distribution statistics and all samples in the present study were reproducible. Scatterplot graphs (Fig. 1E) and a dendrogram (Fig. 1F) demonstrated differences in gene expression of all microarray probe distribution statistics between the KD and the NC group. Differentially expressed genes between 6 samples were identified through fold change filtering.

Statistical analysis. Statistical analysis was performed using SPSS 22.0 (IBM Corp., Armonk, NY, USA). Comparisons between two groups were performed using Fisher's exact test,
Student's t-test and one-way analysis of variance followed by Tukey's honest significant difference test. $\mathrm{P}<0.05$ or $\mathrm{P}<0.01$ was considered to indicate a statistically significant difference. The data are presented as the mean \pm standard deviation.

\section{Results}

ARIDIA mutation in the HEC-1-A cell line and knockdown of mutated ARIDIA by lentiviral shRNA. We first used exon capture sequencing to determine the ARID1A mutation frequencies in the HEC-1-A cell line and observed that all the ARID1A mutations were heterozygous: i) Missense mutations, transversion of $\mathrm{A}$ to $\mathrm{T}$ at codon 1212 on exon 2 and transversion of $\mathrm{G}$ to $\mathrm{T}$ at codon 5281 on exon 20; ii) synonymous mutations, transversion of $\mathrm{C}$ to $\mathrm{A}$ at codon 3969 on exon 16; or iii) nonsense mutations, transversion of $\mathrm{C}$ to $\mathrm{T}$ at codon 5503 on exon 20 (Fig. 2A).

To explore the association of mutated ARID1A with the behavior of EC cell lines, 3 pairs of ARID1A gene shRNA interference fragments; LV-ARID1A-RNA interference (i)-24485-1 (RNAi-1), LV-ARID1A-RNAi-24486-1 (RNAi-2) and LV-ARID1A-RNAi-24487-1 (RNAi-3), one negative control (NC) pair and one blank control (MOCK, empty vector-transfected cells) pair were designed and transfected into HEC-1-A cells. RT-qPCR was used to detect the expression of ARID1A mRNA transfected with interference fragments. ARID1A mRNA expression in HEC-1-A cells following transfection with RNAi-1 ( $\mathrm{P}=0.0042)$, RNAi-2 $(\mathrm{P}=0.0006)$ and RNAi-3 ( $\mathrm{P}=0.0428)$ was significantly decreased compared with that in the NC group. Three shRNA vectors suppressed ARID1A mRNA expression by 78.8, 55.0 and 55.6\%, respectively, compared with that in the NC group. RNAi-1, with an increased transfection efficiency (78.8\%) compared with the $\mathrm{NC}$ group, was selected for subsequent experiments as the KD group (Fig. 2B). Since the ARID1A gene shRNA interference fragment was designed based on codon 6795-6813, it did not fall on the mutation position as mentioned earlier. Therefore, mutated ARID1A may have been depleted by shARID1A knockdown plasmids in the KD group.

Gene expression profiles in response to mutated ARIDIA knockdown by microarray pathway analysis. A total of 408 canonical signaling pathways, 39 disease states and functions, 25 networks, 1,085 molecular targets, 662 upstream and 512 downstream genes were identified in ARID1A-depleted HEC-1-A cells compared with control cells. A Z-score/fold change $\geq 1.0$ represented an activated signaling pathway and disease functions, while a Z-score/fold change $\leq-1.0$ represented an inhibited signaling pathway and disease functions. Furthermore, a signal Z-score/fold change $\geq 1.5$ or $\leq-1.5$ and $\mathrm{P}<0.05$ indicated a significant change in signaling pathways and disease functions between the KD and NC groups.

To investigate specific signaling pathways and functional gene groups, IPA tools were used to identify 143 differentially expressed canonical pathways by defining the enrichment P-value of the pathway using Fisher's exact test $\leq 0.05$, among which 17 pathways were significantly downregulated $(Z$-score $\leq-1.5 ; P<0.05)$ and 13 were significantly upregulated $(\mathrm{Z}$-score $\geq 1.0 ; \mathrm{P}<0.05)$ in the $\mathrm{KD}$ group compared with those in the NC group. The extracellular 
A

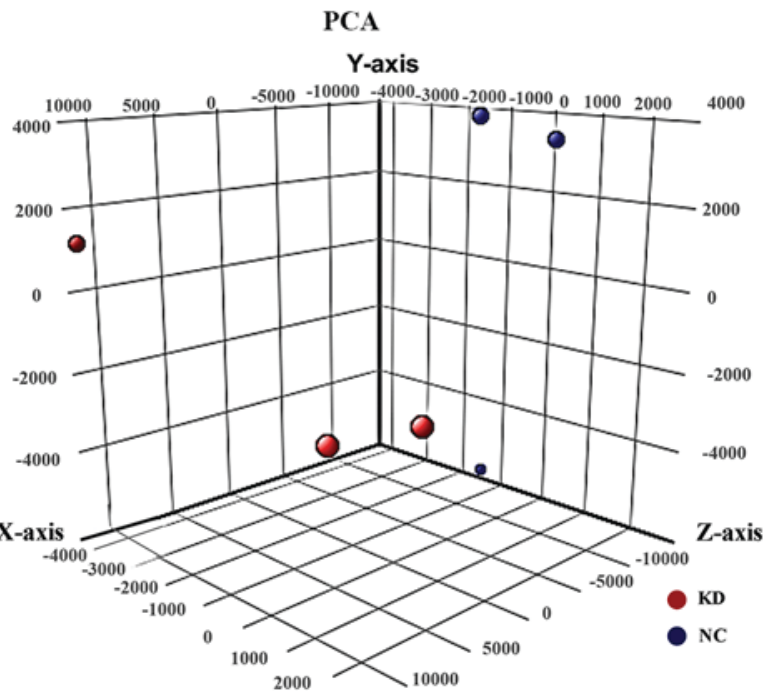

C

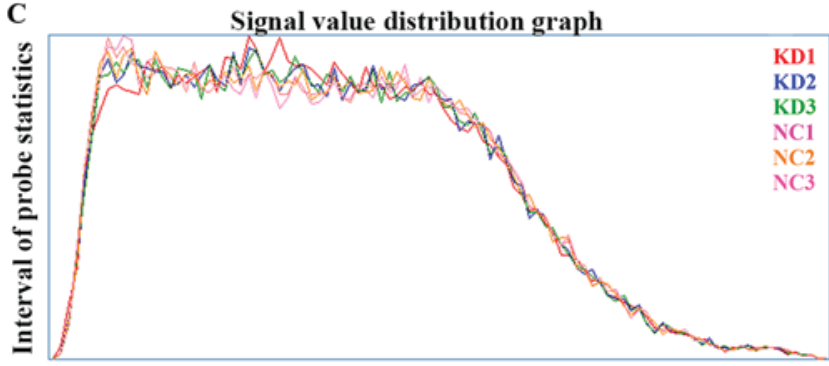

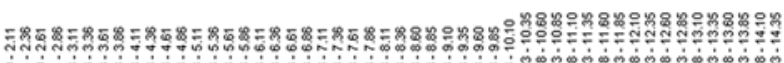

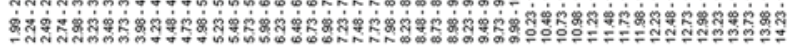
Probe values range
B

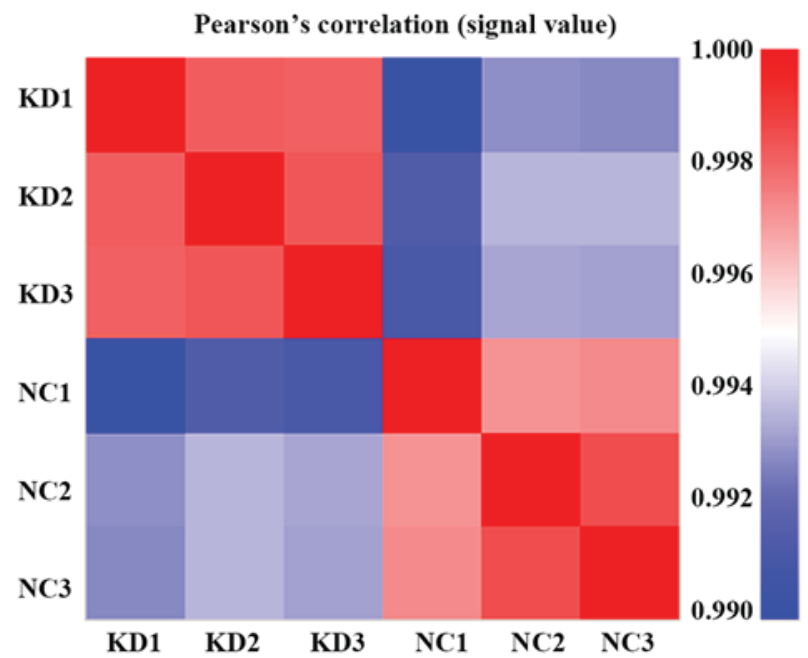

D

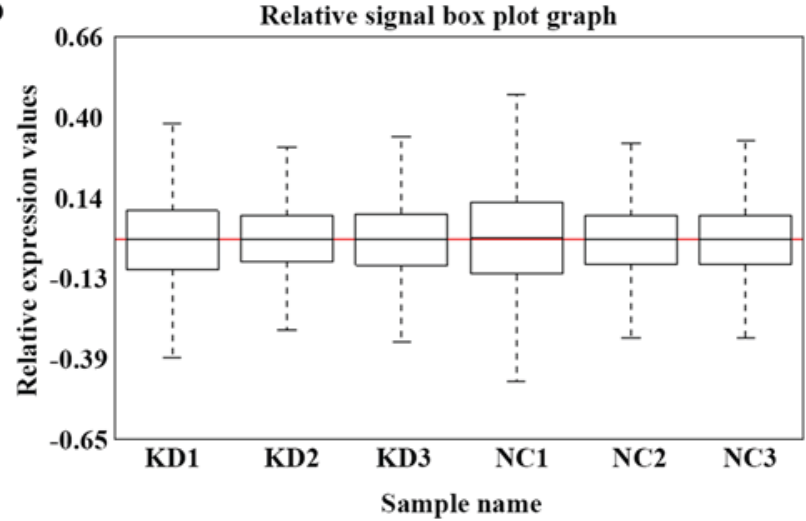

$\mathbf{E}$

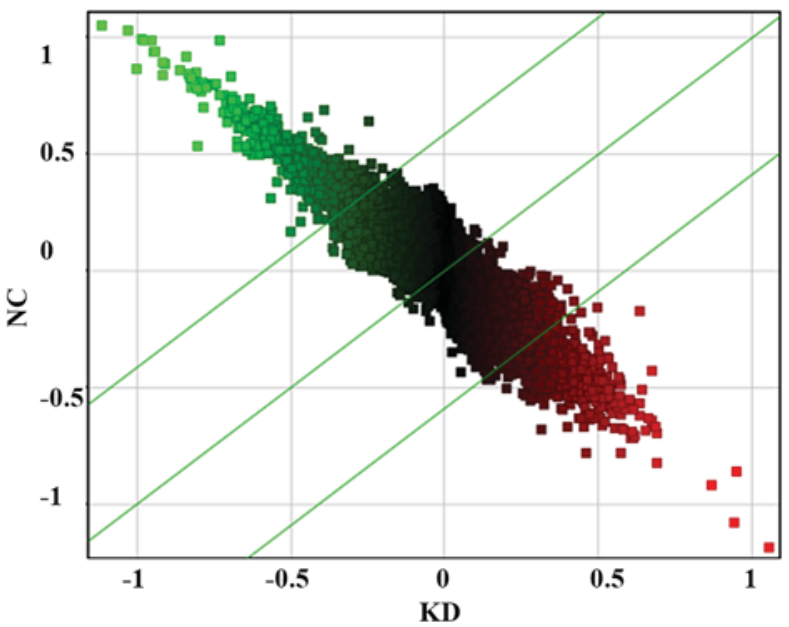

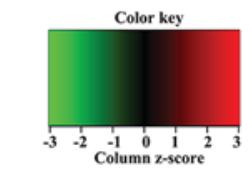

Dendrogram

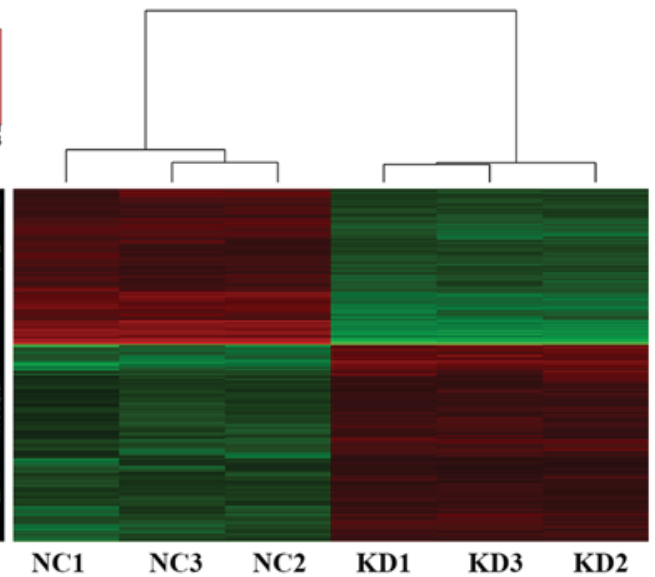

Figure 1. The distributions of 6 samples. (A) PCA graph. Each point represents one KD or NC sample with increased degrees of aggregation between samples indicating more similarity. The axes indicate probes recombined with new synthetic variables, with values representing the score of the weight of the sample in the dimension. Within the groups, the NC group exhibited increased degrees of similarity compared with those of the KD group and there were differences between the KD group and the NC group. (B) Pearson's correlation coefficient (signal value) graph: Each box represents the correlation between the samples on the $\mathrm{x}$-axis and those on the y-axis. Correlation coefficients near to 1.0 correspond to increased signal values of all the chips that are associated with each other. The 6 samples compared in the present study exhibited increased degrees of correlation. (C) Signal value distribution graph. Each curve represents the different values of chips in different probe value ranges. The higher the coincidence degree of signal value distribution curves were, the higher the reliability of the microarray experiments. (D) Relative signal box plot graph: The red line represents the mean expression value of all the samples. The upper and lower horizontal borders of the boxes represent the $90 \%$ confidence intervals and the middle black line represents the median background signal level expression value for each sample. The smaller the range between the upper and lower box edges, the more reliable the relative expression values. (E) Scatterplot graph. The upper portion above the upper green line represents downregulated probes of the KD group and the lower portion below the lower green line represents upregulated probes of the KD group compared with the control group. The middle green line indicates a fold-change of 1. (F) Dendrogram. Each small box represents 1 gene and its color indicates the gene expression level. Darker colors indicate associations with more diseases and functions. The connecting lines at the top of the tree structure were obtained by hierarchical clustering based on the signal values of all samples and the connecting lines to the left side of the tree structure was obtained based on the signal values of all the differences between genes. Two adjacent genes or samples exhibit a higher similarity compared with the NC group. PCA, principal component analysis; KD, knockdown; NC, negative control. 


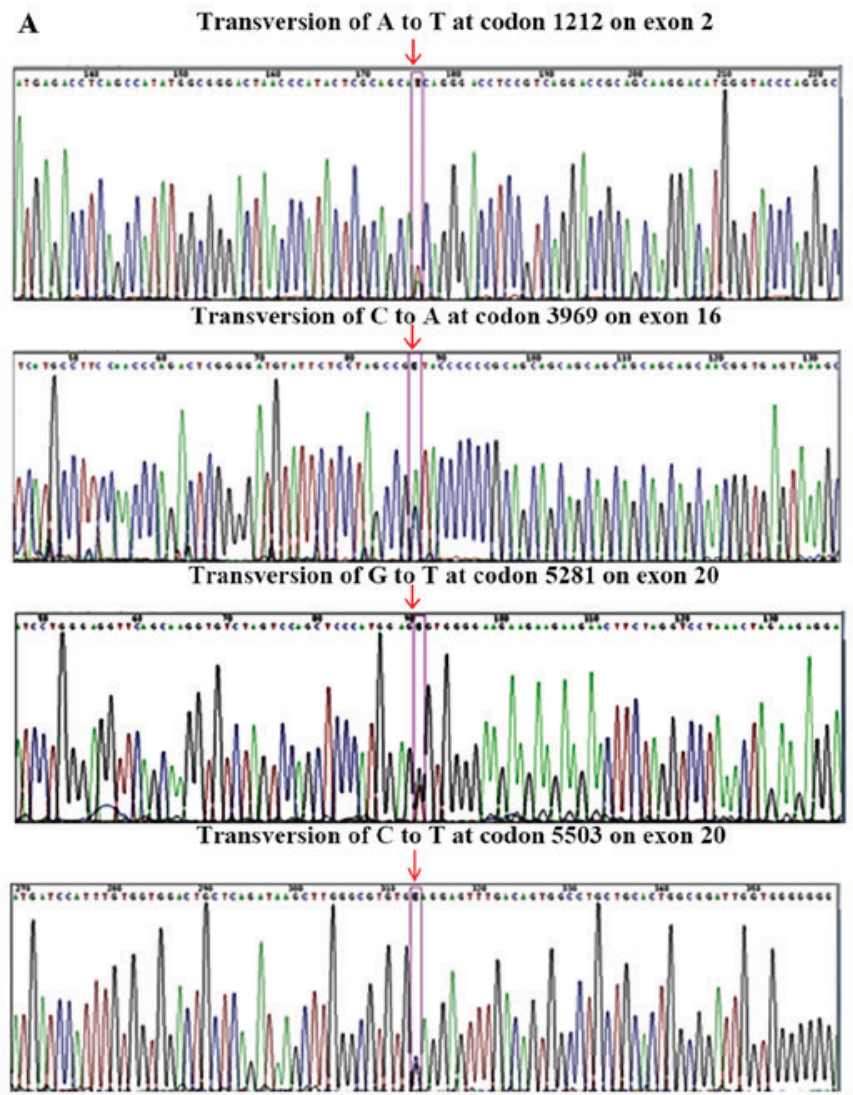

B

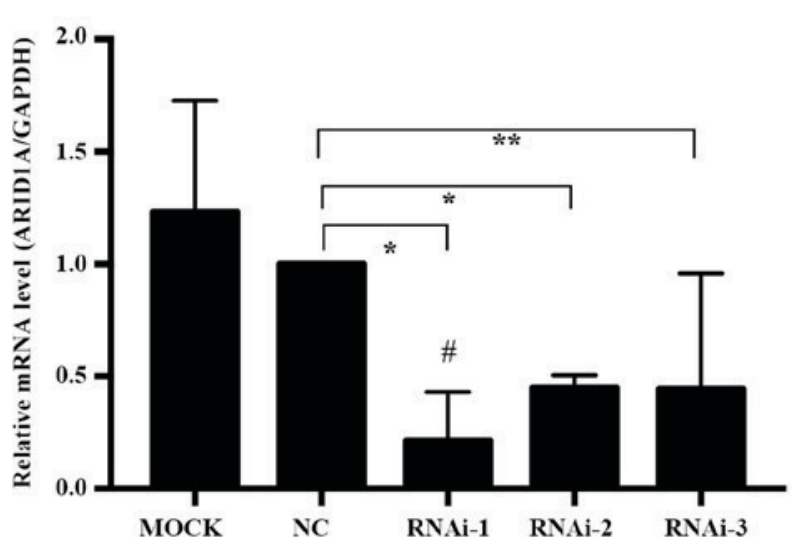

Figure 2. ARID1A mutation in the HEC-1-A cell line and knockdown of mutated ARID1A by lentiviral shRNA. (A) ARID1A heterozygous mutations in the HEC-1-A cell line. (B) The knockdown effects of three lentiviral short hairpin RNA vectors were examined by reverse transcription-quantitative polymerase chain reaction. RNAi-1(\#) yielded the greatest transfection efficiency in HEC-1-A cells $(78.8 \%)$. Data are presented as mean \pm standard deviation. ${ }^{*} \mathrm{P}<0.01,{ }^{* *} \mathrm{P}<0.05$, RNAi vs. NC. ARID1A, AT-rich interactive domain-containing protein $1 \mathrm{~A}$; RNAi, interfering RNA.

signal-regulated kinase $($ ERK)/mitogen-activated protein kinase (MAPK; Z-score=-2.353<-2.0; $\log \mid \mathrm{P}$-valuel=6) and IGF-1 (Z-score=-2.138<-2.0; $\log \mid \mathrm{P}$-valuel=4.06) were the signaling pathways were the 2 most significantly downregulated canonical pathways. Signaling pathways associated with ARID1A-depleted HEC-1-A cells were sequenced by their - $\log (\mathrm{P}$-value $)$. Genes associated with the pathways demonstrating differential expression were highlighted in different colors depending on their $-\log (\mathrm{P}$-value) (Fig. 3A). The ratios presented in Table I represent the ratio of differentially expressed genes from a pathway to the total number of genes of that particular pathway. The majority of these pathways were associated with cancer development, proliferation and apoptosis. The volcano graph (Fig. 3B) demonstrates the distribution of differentially expressed genes by fold change between the KD group and the NC group. Genes presented with a red color indicate $\mathrm{P}<0.05$ and fold change $\geq 1.5$. The heat map demonstrates the differences in 32 genes directly associated with the ERK/MAPK and IGF-1 signaling pathways from the normalized data of 6 experimental groups (Fig. 3C). Microarray analysis indicated that there were 24 significantly downregulated (fold change $\leq-1.5 ; \mathrm{P}<0.05$ ) and 8 significantly upregulated genes (fold change $\geq 1.5 ; \mathrm{P}<0.05$ ) in the KD group compared with the NC group (Fig. 3D).

Gene associated with MAPK/ERK and IGF-1 signaling pathways and significantly affected disease states and functions in response to mutated ARIDIA knockdown. The network diagram of gene interaction analyzed by IPA revealed interactions between ARID1A and molecules associated with the MAPK/ERK and IGF-1 signaling pathways; EIF4E, IGF1R, IRS1 and PIK3CB were downregulated, while FOS and FOXO1 were upregulated (Fig. 4A). To confirm these results, western blot analysis was used to investigate alternations in the activities of the MAPK/ERK and IGF-1 signaling pathways upon ARID1A depletion in HEC-1-A cells. Multiple cellular processes were potentially affected. IRS (Z-score=-1.546), PIK3CB (Z-score=-1.673) and IGF1R $(Z$-score $=-1.952)$ were significantly downregulated in shARID1A-HEC-1-A cells compared with that in control cells. However, ARID1A knockdown significantly elevated the level of FOXO1 $(Z$-score $=1.588)$ and there were no significant differences in EIF4E $(Z$-score $=-1.531)$ or FOS $(Z$-score=1.975) expression in the shARID1A-HEC-1-A cells compared with that in the control cells (Fig. 4B and C).

To evaluate the association of disease states and functions with biological networks, 39 affected disease states and functions were identified. A heat map was produced demonstrating the association between activated or inhibited disease states or functions and different Z-scores in the KD group compared with those of the control group. A total of 9 disease states and functions were significantly upregulated, including carcinoma tumorigenesis, mitosis of tumor cell lines, cancer, adenocarcinoma, tumorigenesis of malignant tumors, mitochondria density, tumor necrosis, tumor cell death and cancer cell death; 5 functions were significantly downregulated, namely cell proliferation, cell survival, uptake of D-glucose, cell viability and tumor growth. The affected disease states and functions graph was constructed based on the -Log (P-value), and genes within the disease states and functions that exhibited differential expression were highlighted in different colors depending on their-Log (P-value) (Fig. 4D; Table II).

\section{Discussion}

In 2012, ARID1A mutations were reported to occur in $41.6 \%$ of EC cases in America (4). The majority of cancer-associated ARID1A mutations (>97\%) are inactivating, with nonsense or frameshift, rather than silent or missense, mutations detected throughout the gene (7). Jones et al (8) has determined that in $30 \%$ of the ovarian clear cell carcinomas with ARID1A 
Table I. Significantly affected canonical signaling pathways in AT-rich interactive domain-containing protein 1A-depleted HEC-1-A cells

\begin{tabular}{|c|c|c|c|c|}
\hline Signaling pathway & -Log(P-value $)$ & Ratio & Z-score & Molecules \\
\hline $\begin{array}{l}\text { Extracellular signal-regulated } \\
\text { kinase/mitogen-activated protein } \\
\text { kinase }\end{array}$ & 6 & 0.141 & -2.353 & $\begin{array}{l}\text { PRKACB, PPP2R2A, SOS2, CRK, EIF4E, LAMTOR3, } \\
\text { PPP1CC, ELF3, PPP1R12A, PPP1R10, PPP2R2C, } \\
\text { ATM, PIK3C2A, YWHAZ, MKNK2, RAP1A, ATF2, } \\
\text { FOS, PRKCI, PRKAR2B, IRS1, PAK2, PRKAG2, } \\
\text { PIK3CB, RPS6KA1, ELK3, PPP2R1B, PRKAR1A }\end{array}$ \\
\hline Insulin-like growth factor 1 & 4.06 & 0.151 & -2.138 & $\begin{array}{l}\text { PRKACB, NEDD4, PIK3C2A, SOS2, YWHAZ, } \\
\text { FOS, PRKCI, PRKAR2B, FOXO1, IRS1, IGFBP3, } \\
\text { PRKAG2, IGF1R, PIK3CB, PRKAR1A, ATM }\end{array}$ \\
\hline Cyclin-dependent kinase 5 & 3.33 & 0.141 & -2.138 & $\begin{array}{l}\text { PRKACB, PPP1CC, PRKAR2B, PPP1R 10, } \\
\text { PPP1R12A, PPP2R2A, EGR1, PRKAG2, MAPK10, } \\
\text { LAMA1, MAPK9, PPP2R2C, PPP2R1B, PRKAR1A }\end{array}$ \\
\hline $\begin{array}{l}\text { Inhibition of angiogenesis by } \\
\text { thrombospondin } 1\end{array}$ & 2.86 & 0.206 & 2 & $\begin{array}{l}\text { MAP2K4, TGFBR2, GUCY1A3, SDC2, MAPK10, } \\
\text { MAPK9, NOS3 }\end{array}$ \\
\hline $\begin{array}{l}\text { Activation of interferon regulatory } \\
\text { factor by cytosolic pattern } \\
\text { recognition receptors }\end{array}$ & 1.43 & 0.113 & 1.89 & $\begin{array}{l}\text { MAP2K4, TANK, DDX58, SIKE1, MAPK10, MAPK9, } \\
\text { ATF2 }\end{array}$ \\
\hline $\begin{array}{l}\text { Dopamine-DARPP32 feedback in } \\
\text { cAMP signaling }\end{array}$ & 2.08 & 0.0988 & -3.051 & $\begin{array}{l}\text { PRKACB, GUCY1A3, PPP2R2A, KCNJ16, CSNK1A1, } \\
\text { ATF2, PPP1CC, PLCB4, PRKCI, PRKAR2B, } \\
\text { PPP1R12A, PPP1R10, PRKAG2, PPP2R2C, PPP2R1B, } \\
\text { PRKAR1A }\end{array}$ \\
\hline $\begin{array}{l}\text { P2Y purigenic receptor signaling } \\
\text { pathway }\end{array}$ & 1.78 & 0.0985 & -2.496 & $\begin{array}{l}\text { PRKACB, PIK3C2A, ATF2, ITGB3, FOS, PLCB4, } \\
\text { PRKCI, PRKAR2B, IRS1, PRKAG2, PIK3CB, } \\
\text { PRKAR1A, ATM }\end{array}$ \\
\hline Synaptic long-term potentiation & 1.72 & 0.1 & -2.887 & $\begin{array}{l}\text { PRKACB, PLCB4, PPP1CC, PRKCI, PRKAR2B, } \\
\text { PPP1R10, PPP1R12A, PRKAG2, RPS6KA1, RAP1A, } \\
\text { PRKAR1A, ATF2 }\end{array}$ \\
\hline Endothelial nitric oxide signaling & 1.58 & 0.0903 & -2.138 & $\begin{array}{l}\text { PRKACB, PIK3C2A, GUCY1A3, HSPA1A/HSPA1B, } \\
\text { NOS3, HSPA2, PRKCI, PRKAR2B, IRS1, PRKAG2, } \\
\text { PIK3CB, CASP8, PRKAR1A, ATM }\end{array}$ \\
\hline $\begin{array}{l}\text { Melanocyte development and } \\
\text { pigmentation signaling }\end{array}$ & 3.52 & 0.147 & -2.138 & $\begin{array}{l}\text { PRKACB, PTPN6, PIK3C2A, SOS2, CRK, ATF2, } \\
\text { RPS6KA6, PRKAR2B, IRS1, PRKAG2, PIK3CB, } \\
\text { RPS6KA1, PRKAR1A, ATM }\end{array}$ \\
\hline $\begin{array}{l}\text { Neuropathic pain signaling in } \\
\text { dorsal horn neurons }\end{array}$ & 1.52 & 0.0965 & -2.111 & $\begin{array}{l}\text { PRKACB, FOS, PLCB4, PRKCI, PRKAR2B, PIK3C2A, } \\
\text { IRS1, PRKAG2, PIK3CB, ATM, PRKAR1A }\end{array}$ \\
\hline $\begin{array}{l}\text { Nitric oxide signaling in the } \\
\text { cardiovascular system }\end{array}$ & 1.54 & 0.0973 & -2.111 & $\begin{array}{l}\text { PRKACB, PRKCI, PRKAR2B, GUCY1A3, PIK3C2A, } \\
\text { IRS1, PRKAG2, PIK3CB, NOS3, ATM, PRKAR1A }\end{array}$ \\
\hline
\end{tabular}

DARPP32, cAMP-regulated neuronal phosphoprotein.

mutations, in wild-type and mutant alleles, both alleles were affected, suggesting that post-transcriptional mechanisms account for loss of ARID1A protein in ovarian clear cell carcinomas harboring heterozygous mutations without loss of heterozygosity (7). In a previous study, immunohistochemistry (IHC) revealed that $27 \%$ of ARID1A heterozygous ovarian clear cell carcinomas retained detectable protein expression, as did $5 \%$ of tumors not found to possess coding mutations (7). In addition, $25 \%$ of gastric cancer cases harboring heterozygous mutations retained ARID1A expression, as determined by IHC (13). Therefore, mutations affecting ARID1A expression may occur in non-coding regions of the genome not assayed by exome sequencing techniques and epigenetic silencing may contribute to this.

In the present study, the HEC-1-A cell line was selected to exclude the effects of PTEN mutations and estrogen (ER) since the HEC-1-A cell line expresses negative or low ER and carries wild-type PTEN (14). The present study determined heterozygous ARID1A mutations in the HEC-1-A cell line, suggesting that biologically relevant haploin sufficient effects were caused by loss of a single allele. Another aim of the present study was to elucidate the mechanisms that mediate 
A

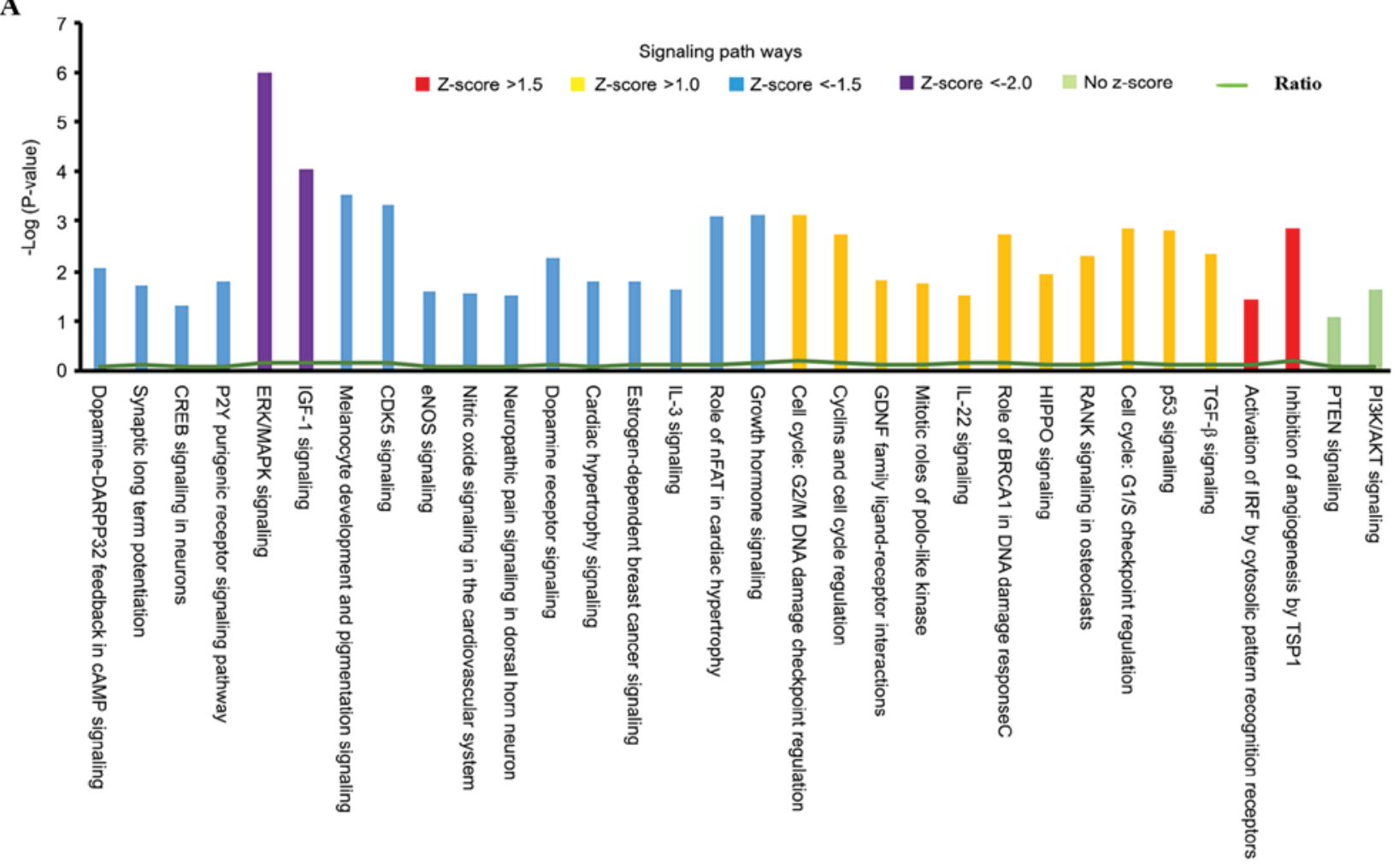

B

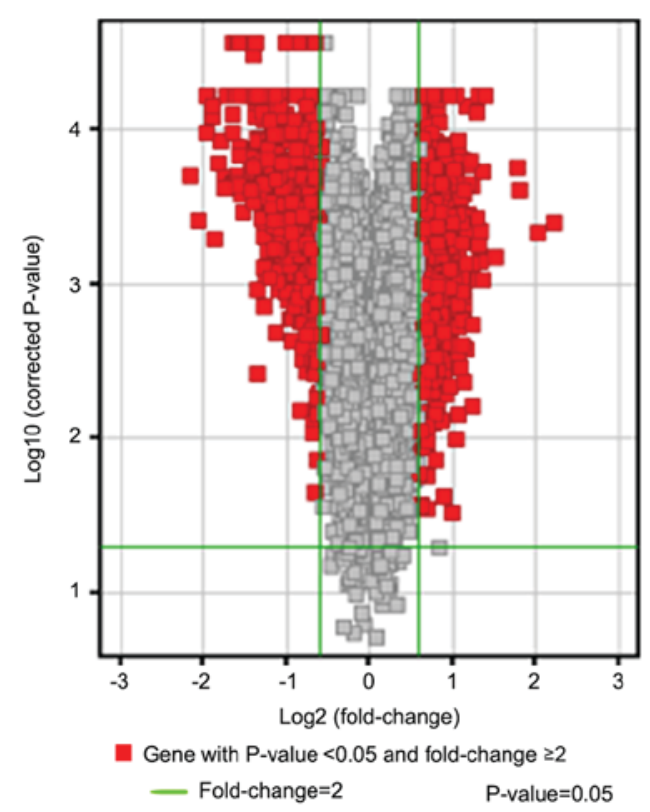

C

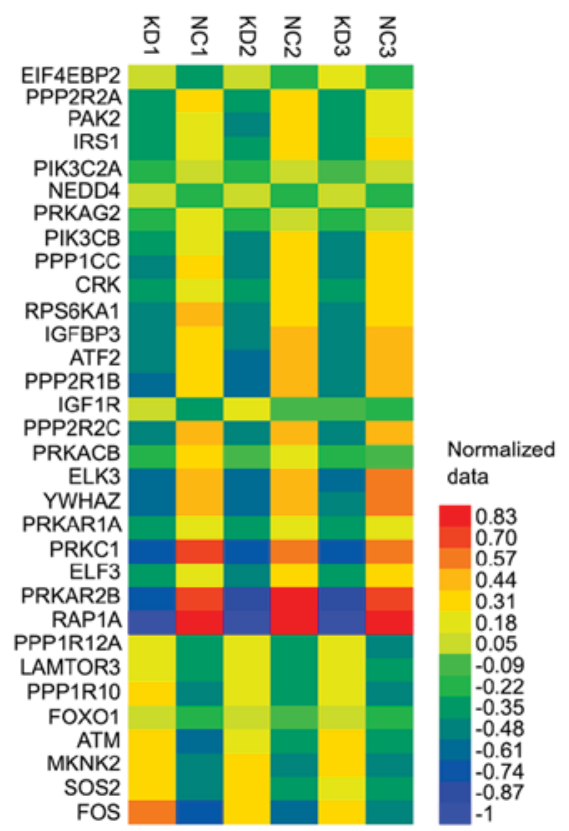

D

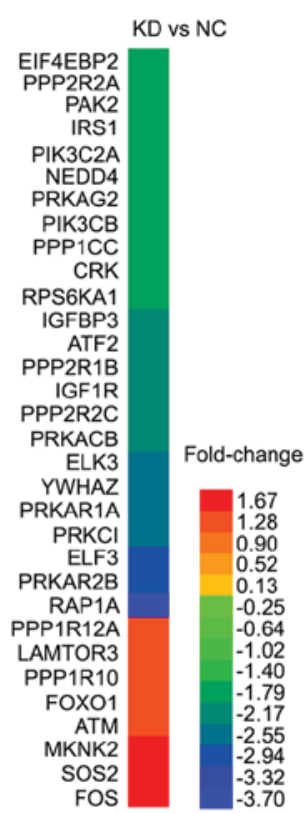

Figure 3. Signaling pathways and heat map of specific genes. (A) Signaling pathways graph. The ratio represents differentially expressed genes from a pathway to the total number of genes of that particular pathway. (B) Volcano graph of all differentially expressed genes. (C) Normalized data of 32 genes from 6 experimental groups. (D) Heat map of 32 specific genes of the mitogen-activated protein kinase/extracellular signal-regulated kinase and insulin growth factor-1 signaling pathways in ARID1A-depleted HEC-1-A cells compared with those in the control group. KD, knockdown; NC, negative control; ARID1A, AT-rich interactive domain-containing protein 1A.

the effects of ARID1A mutations on type I EC by performing complemented gene- and pathway-focused studies. A microarray-based study was performed to reveal numerous associated signaling pathways and processes, including those associated with cell proliferation, apoptosis and metabolism. It was hypothesized that the MAPK/ERK and IGF-1 signaling pathways were the 2 most significantly downregulated pathways in the mutated ARID1A-depleted EC cell line based on the results of a microarray-based study and IPA analysis. Thus far, few studies have been reported regarding the mechanism of ARID1A mutations, via the MAPK/ERK and IGF-1 signaling pathways, in the development of EC.

Type IEC is commonly regarded as a metabolic syndromeassociated tumor, characterized by insulin resistance and 
Table II. Significantly affected disease states and functions based on - $\log (\mathrm{P}-\mathrm{value})$ in AT-rich interactive domain-containing protein 1A-depleted HEC-1-A cells.

\begin{tabular}{llcr}
\hline Categories & \multicolumn{1}{c}{ Disease states and functions } & -Log(P-value) & Z-score \\
\hline Cancer; organismal injury and abnormalities & Tumorigenesis of carcinoma & 4.169 & 2.577 \\
Cancer; organismal injury and abnormalities & Cancer & 14.22 & 1.98 \\
Cancer; organismal injury and abnormalities & Adenocarcinoma & 14.15 & 1.835 \\
Cell death and survival & Cell survival & 10.12 & -1.678 \\
Cell death and survival & Cell viability & 9.90 & -1.609 \\
Cancer; organismal injury and abnormalities & Tumorigenesis of malignant tumor & 6.104 & 1.818 \\
Cellular growth and proliferation & Proliferation of cells & 4.91 & -2.016 \\
Cancer; organismal injury and abnormalities & Growth of tumor & 4.54 & -1.52 \\
$\begin{array}{l}\text { Cellular assembly and organization } \\
\text { Cancer; cell death and survival; organismal injury and }\end{array}$ & Density of mitochondria & 4.45 & 1.746 \\
abnormalities; tumor morphology & Necrosis of tumor & 4.32 & 1.553 \\
$\begin{array}{l}\text { Cancer; cell death and survival; organismal injury and } \\
\text { abnormalities; tumor morphology }\end{array}$ & Death of tumor cells & 4.32 & 1.553 \\
$\begin{array}{l}\text { Cancer; cell death and survival; organismal injury and } \\
\text { abnormalities; tumor morphology }\end{array}$ & Death of cancer cells & 2.88 & 1.52 \\
$\begin{array}{l}\text { Carbohydrate metabolism; molecular transport; small } \\
\text { molecule biochemistry }\end{array}$ & Uptake of D-glucose & 2.63 & -1.625 \\
Cell cycle & Mitosis of tumor cell lines & 2.51 & 2.236 \\
\hline
\end{tabular}

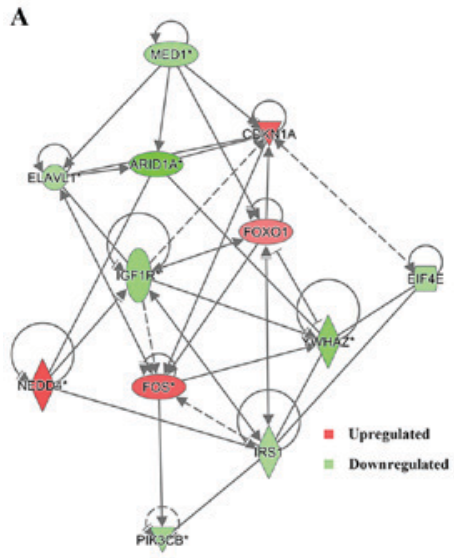

B
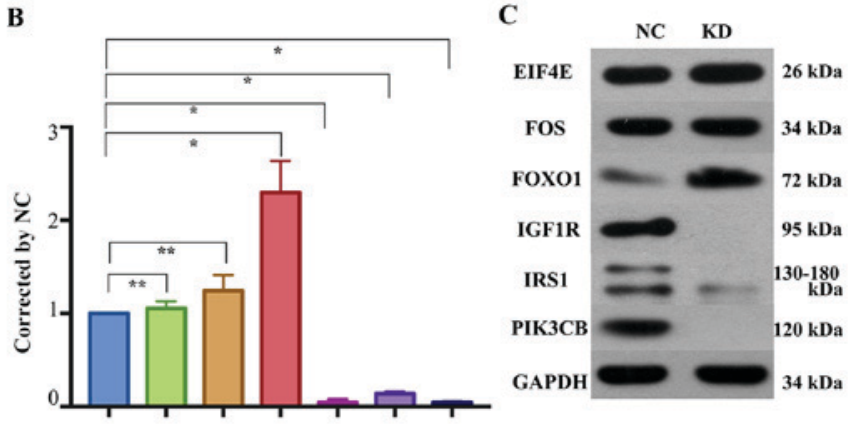

NC $\square$ EIF4E $\square$ FOS FOXO

IGF1R IRS1 $\square$ PIK3CB

D

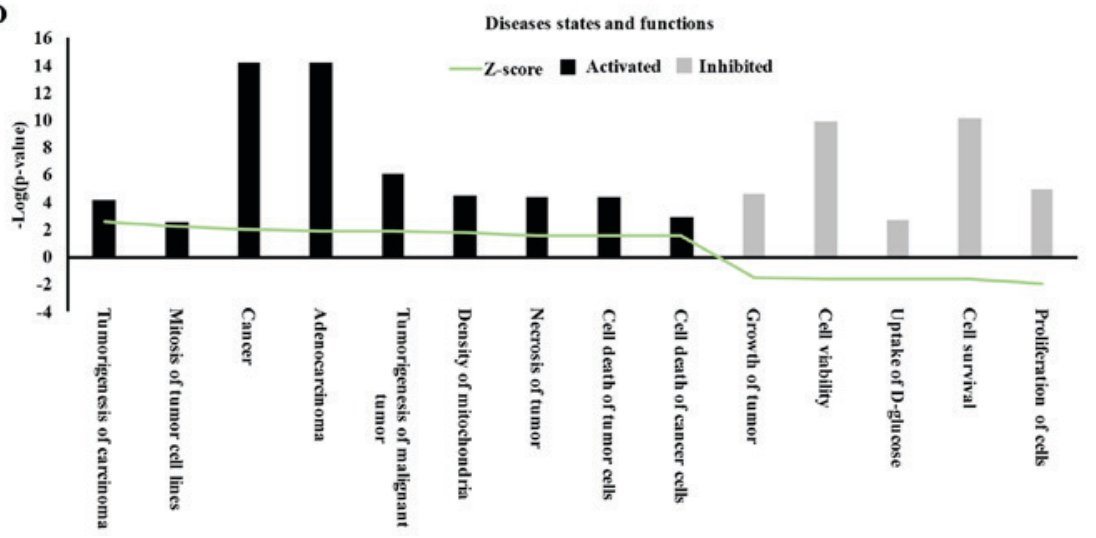

Figure 4. Gene associated with MAPK/ERK and IGF-1 signaling pathways and significantly affected disease states and functions in response to mutated ARID1A knockdown. (A) Network graph of the association between ARID1A and genes associated with the mitogen-activated protein kinase/extracellular signal-regulated kinase and insulin growth factor-1 signaling pathways. FOXO1 was significantly increased; IRS1, PIK3CB and IGF1R were significantly decreased; and EIF4E and Fos were not significantly altered by ARID1A knockdown, compared with the control cells, as (B) determined by western blot analysis and (C) quantified by the peak area of triplicate experiments. GAPDH was used as a loading control. Data are presented as mean \pm standard deviation. ${ }^{*} \mathrm{P}<0.01, \mathrm{KD}$ vs. NC. ${ }^{* *} \mathrm{P}<0.05$. (D) Heat map of significantly activated or inhibited diseases and functions associated with short hairpin ARID1A-HEC-1-A cells, as determined by sequencing of the - $\log (\mathrm{P}-\mathrm{value})$. ARID1A, AT-rich interactive domain-containing protein 1A; FOXO1, forkhead box protein O1; IRS1, insulin receptor substrate 1; PIK3CB, phosphatidylinositol-4,5-bisphosphate 3-kinase catalytic subunit $\beta$; IGF1R, insulin-like growth factor 1 receptor; EIF4E, eukaryotic translation initiation factor 4E. 
associated with tumor occurrence $(15,16)$. A previous study demonstrated that insulin was revealed to stimulate the proliferation and migration of EC cells and to inhibit their apoptosis through the IGF-1 signaling pathway (17). IRS1 is an upstream component of the MAPK/ERK and the IGF-1 signaling pathways and is associated with a susceptibility to insulin resistance. Global activation of IGF-IR signaling and loss of negative feedback to IRS-1 appear to be reprogrammed in endometrial hyperplasia (18). IGF1R is overexpressed in the majority of malignant tissues, where it binds IGF to function as an anti-apoptotic agent by enhancing cell survival $(19,20)$. In the present study, downregulation of IRS1 and IGF1R was detected upon ARID1A depletion in HEC-1-A cell lines.

However, certain patients with combined EC and obesity do not exhibit hyperinsulinemia, implying that other factors are also associated with the occurrence and development of EC. IGF1 and IGF2 are strong mitogens that exert proliferative and anti-apoptotic effects via 2 downstream signaling pathways, the MAPK/ERK and phosphatidylinositol-4,5-bisphosphate 3-kinase (PI3K)/protein kinase B cascades (2). The IR, IRS1/2 and MAPK/ERK1/2 signaling pathways have all been revealed to be highly expressed in EC (19,21). Wang et al (21) demonstrated that visfatin stimulated EC cell proliferation by inducing G1/S cycle progression via activation of IR, PI3K/protein kinase B and MAPK/ERK1/2 signaling pathways and consequent upregulation of MYC proto-oncogene, bHLH transcription factor and cyclin D1. A previous study involving 6 EC cell lines also revealed that the direct mechanism involves the activation of Ras/MAPK signaling pathway crosstalk in EC via the function of insulin, IGF-1 and ER (22).

It has been indicated that the IGF-1 and MAPK/ERK pathways are able to interact with one another (23). The IGF-1 pathway has been revealed to modulate cell apoptosis, while the MAPK/ERK pathway has been implicated in cell proliferation (2). The IGF-1R signaling pathway is initiated upon the binding of IGF-1 to its cell-surface IGF-1R to activate the MAPK/ERK signaling pathway (24). In addition, upon insulin-binding, the IR is activated, triggering activation of IRS-1, which then activates the PI3K and MAPK pathways to stimulate cell growth and proliferation and to inhibit programmed cell death (24-26).

$\mathrm{PI} 3 \mathrm{KC} \beta$ has been revealed to be part of the upstream gene in the MAPK/ERK and IGF-1 signaling pathways (27). FOXO1, a downstream gene in the IGF-1 signaling pathway that is usually downregulated in endometrial tumor tissues and cell lines (28), is able to regulate cell growth and differentiation. In the present study, the downregulation of PIK3C $\beta$ and the overexpression of FOXO1 was observed in ARID1A-knockdown transfected cell lines.

The present study also established that carcinoma tumorigenesis, mitosis of tumor cell lines, tumorigenesis of malignant tumors, tumor cell death and cancer cell death were significantly upregulated disease states and functions according to IPA, while the proliferation of cells, cell survival and tumor growth were significantly downregulated.

To conclude, our biochemical microarray analysis demonstrated 13 upregulated and 17 downregulated pathways and 14 significantly affected disease states and functions in mutated ARID1A-depleted HEC-1-A cells. To the best of our knowledge, the present study was also the first to demonstrate a potential association between ARID1A mutations and EC development that involved sequential activation of the MAPK/ERK and IGF-1 signaling pathways through activation of IRS1, PIK3CB and IGF1R and inhibition of FOXO1. The results of the present study provide an opportunity to identify novel therapeutic targets based on the proposed function for ARID1A mutations in tumor progression in EC. However, elucidating the underlying mechanisms requires further investigation.

\section{Acknowledgements}

The present study was supported by the Key Project for Major Diseases of Health System of Shanghai Municipality (grant no. 2013ZYJB0201).

\section{References}

1. Siegel RL, Miller KD and Jemal A: Cancer statistics, 2015. CA Cancer J Clin 65: 5-29, 2015.

2. Wang Y, Zhu Y, Zhang L, Tian W, Hua S, Zhao J, Zhang H and Xue F: Insulin promotes proliferation, survival, and invasion in endometrial carcinoma by activating the MEK/ERK pathway. Cancer Lett 322: 223-231, 2012.

3. de Boer SM, Powell ME, Mileshkin L, Katsaros D, Bessette P, Haie-Meder C, Ottevanger PB, Ledermann JA, Khaw P, Colombo A, et al: Toxicity and quality of life after adjuvant chemoradiotherapy versus radiotherapy alone for women with high-risk endometrial cancer (PORTEC-3): An open label, multicentre, randomised, phase 3 trial. Lancet Oncol 17: 1114-1126, 2016.

4. Liang H, Cheung LW, Li J, Ju Z, Yu S, Stemke-Hale K, Dogruluk T, Lu Y, Liu X, Gu C, et al: Whole-exome sequencing combined with functional genomics reveals novel candidate driver cancer genes in endometrial cancer. Genome Res 22: 2120-2129, 2012

5. Mao TL, Ardighieri L, Ayhan A, Kuo KT, Wu CH, Wang TL and Shih IeM: Loss of ARID1A expression correlates with stages of tumor progression in uterine endometrioid carcinoma. Am J Surg Pathol 37: 1342-1348, 2013.

6. Er TK, Su YF, Wu CC, Chen CC, Wang J, Hsieh TH, Herreros-Villanueva M, Chen WT, Chen YT, Liu TC, et al: Targeted next-generation sequencing for molecular diagnosis of endometriosis-associated ovarian cancer. J Mol Med (Berl) 94: 835-847, 2016.

7. Wiegand KC, Shah SP, Al-Agha OM, Zhao Y, Tse K, Zeng T, Senz J, McConechy MK, Anglesio MS, Kalloger SE, et al: ARID1A mutations in endometriosis-associated ovarian carcinomas. N Engl J Med 363: 1532-1543, 2010.

8. Jones S, Wang TL, Shih IeM, Mao TL, Nakayama K, Roden R, Glas R, Slamon D, Diaz LA Jr, Vogelstein B, et al: Frequent mutations of chromatin remodeling gene ARID1A in ovarian clear cell carcinoma. Science 330: 228-231, 2010.

9. Zhai Y, Kuick R, Tipton C, Wu R, Sessine M, Wang Z, Baker SJ, Fearon ER and Cho KR: Aridla inactivation in an Apc- and Pten-defective mouse ovarian cancer model enhances epithelial differentiation and prolongs survival. J Pathol 238: 21-30, 2016

10. Ayhan A, Mao TL, Seckin T, Wu CH, Guan B, Ogawa H, Futagami M, Mizukami H, Yokoyama Y, Kurman RJ and Shih IeM: Loss of ARID1A expression is an early molecular event in tumor progression from ovarian endometriotic cyst to clear cell and endometrioid carcinoma. Int J Gynecol Cancer 22: 1310-1315, 2012.

11. $\mathrm{Wu} \mathrm{JN}$ and Roberts CW: ARID1A mutations in cancer: Another epigenetic tumor suppressor? Cancer Discov 3: 35-43, 2013.

12. Arocho A, Chen B, Ladanyi M and Pan Q: Validation of the 2-DeltaDeltaCt calculation as an alternate method of data analysis for quantitative PCR of BCR-ABL P210 transcripts. Diagn Mol Pathol 15: 56-61, 2006.

13. Zang ZJ, Cutcutache I, Poon SL, Zhang SL, McPherson JR, Tao J, Rajasegaran V, Heng HL, Deng N, Gan A, et al: Exome sequencing of gastric adenocarcinoma identifies recurrent somatic mutations in cell adhesion and chromatin remodeling genes. Nat Genet 44: 570-574, 2012. 
14. Gagnon V, Mathieu I, Sexton E, Leblanc K and Asselin E: AKT involvement in cisplatin chemoresistance of human uterine cancer cells. Gynecol Oncol 94: 785-795, 2004.

15. Trabert B, Wentzensen N, Felix AS, Yang HP, Sherman ME and Brinton LA: Metabolic syndrome and risk of endometrial cancer in the United States: A study in the SEER-medicare linked database. Cancer Epidemiol Biomarkers Prev 24: 261-267, 2015.

16. Stocks T, Bjørge T, Ulmer H, Manjer J, Häggström C, Nagel G, Engeland A, Johansen D, Hallmans G, Selmer R, et al: Metabolic risk score and cancer risk: Pooled analysis of seven cohorts. Int J Epidemiol 44: 1353-1363, 2015.

17. Wang Y, Hua S, Tian W, Zhang L, Zhao J, Zhang H, Zhang W and Xue F: Mitogenic and anti-apoptotic effects of insulin in endometrial cancer are phosphatidylinositol 3-kinase/Akt dependent. Gynecol Oncol 125: 734-741, 2012.

18. McCampbell AS, Walker CL, Broaddus RR, Cook JD and Davies PJ: Developmental reprogramming of IGF signaling and susceptibility to endometrial hyperplasia in the rat. Lab Invest 88: 615-626, 2008.

19. Le Roith D: The insulin-like growth factor system. Exp Diabesity Res 4: 205-212,2003

20. Bruchim I, Sarfstein R, Reiss A, Flescher E and Werner H: IGF1R tyrosine kinase inhibitor enhances the cytotoxic effect of methyl jasmonate in endometrial cancer. Cancer Lett 352: 214-219, 2014

21. Wang Y, Gao C, Zhang Y, Gao J, Teng F, Tian W, Yang W, Yan Y and Xue F: Visfatin stimulates endometrial cancer cell proliferation via activation of PI3K/Akt and MAPK/ERK1/2 signalling pathways. Gynecol Oncol 143: 168-178, 2016.
22. Ogawa K, Sun C and Horii A: Exploration of genetic alterations in human endometrial cancer and melanoma: Distinct tumorigenic pathways that share a frequent abnormal PI3K/AKT cascade. Oncol Rep 14: 1481-1485, 2005.

23. Li Y, Jia Y, Che Q, Zhou Q, Wang K and Wan XP: AMF/PGImediated tumorigenesis through MAPK-ERK signaling in endometrial carcinoma. Oncotarget 6: 26373-26387, 2015.

24. Tao Y, Pinzi V, Bourhis J and Deutsch E: Mechanisms of disease: Signaling of the insulin-like growth factor 1 receptor pathway-therapeutic perspectives in cancer. Nat Clin Pract Oncol 4: 591-602, 2007.

25. Ewing GP and Goff LW: The insulin-like growth factor signaling pathway as a target for treatment of colorectal carcinoma. Clin Colorectal Cancer 9: 219-223, 2010.

26. Tognon CE and Sorensen PH: Targeting the insulin-like growth factor 1 receptor (IGF1R) signaling pathway for cancer therapy. Expert Opin Ther Targets 16: 33-48, 2012.

27. Campbell M, Allen WE, Sawyer C, Vanhasebroeck B and Trimble ER: Glucose-potentiated chemotaxis in human vascular smooth muscle is dependent on cross-talk between the PI3K and MAPK signaling pathways. Circ Res 95: 380-388, 2004.

28. Ward EC, Hoekstra AV, Blok LJ, Hanifi-Moghaddam P, Lurain JR, Singh DK, Buttin BM, Schink JC and Kim JJ: The regulation and function of the forkhead transcription factor, Forkhead box O1, is dependent on the progesterone receptor in endometrial carcinoma. Endocrinology 149: 1942-1950, 2008. 